\title{
ОДЕЛЬ ЗВЕНА СЕТИ ПЕРЕДАЧИ ДАННЫХ С ГРАДИЕНТНЫМ РЕЗЕРВИРОВАНИЕМ КАНАЛЬНОГО РЕСУРСА ДЛЯ ОПИСАНИЯ ПЕРЕДАЧИ МНОГОМОДАЛЬНОЙ ИНФОРМАЦИИ
}

\section{MODEL OF A DATA TRANSMISSION NETWORK LINK WITH GRADIENT REDUNDANCY OF A CHANNEL RESOURCE FOR DESCRIBING THE TRANSMISSION OF MULTIMODAL INFORMATION}

S. Saitov A. Saitov

Summary. The article proposes a new approach to modeling by means of the theory of queuing processes of joint service with reservation of the channel resource of protocol data units of messages of traditional communication services and messages in a multimodal representation in the link of the data transmission network of the monitoring system of critical state facilities.

Keywords: model, data transmission network, critical object, operator identification, monitoring, efficiency of resource use.

\section{Обшие положения}

$\boldsymbol{\Gamma}$ еополитическая обстановка в мире требует существенного ужесточения мониторинга критически важных объектов (КВO) государства [1, 2], так как отказ (повреждение) их элементов может приводить к существенным негативным военно-политическим и экономическим последствиям, а иногда к многочисленным человеческим жертвам [3]. Для снижения влияния «человеческого фактора» в КВО широко внедряются средства мониторинга поведения операторов автоматизированных рабочих мест (АРМ). Перспективными для автоматической идентификации легитимности и девиантности персонала КВО признаны системы на базе динамической многомодальной аутентификации (ДМА) [4]. Внедрение новых средств ДМА приводит к увеличению нагрузки на соответствующие сети передачи данных (СПД), где наряду с данными контроля передаются сообщения традиционных услуг связи (ТУС). Кроме того, поток сообщений модальностей обладают рядом специфических характеристик, не учет которых при обслуживании в СПД приведет к недостоверным выводам о состоянии операторов АРM [5]. В следствие изложенного актуальной научно-технической задачей предметной области является моделирование процессов совмест-

\author{
Саитов Сергей Игоревич \\ Сотрудник, Академия ФСО России, г. Орел \\ soul1308@yandex.ru \\ Саитов Андрей Игоревич \\ Сотрудник, Академия ФСО России, г. Орел
}

Аннотация. В статье предлагается новый подход к моделированию средствами теории массового обслуживания процессов совместного обслуживания с резервированием канального ресурса протокольных блоков данных сообщений традиционных услуг связи и сообщений в многомодальном представлении в звене сети передачи данных системы мониторинга критически важных объектов государства.

Ключевые слова: модель, сеть передачи данных, критически важный объект, идентификация оператора, мониторинг, эффективность использования ресурсов.

ного обслуживания протокольных блоков данных (ПБД) сообщений ТУС и сообщений в многомодальном представлении. Ниже такое моделирование предлагается осуществить на основе положений теории массового обслуживания (ТМО) [6-9] для систем с резервированием канального ресурса (РКP).

\section{Постановка заАачи моделирования СП $\triangle$}

Пусть в рассматриваемой СПД даже с учетом внедрения средств ДМА по экономическим соображениям не планируется увеличения объемов канального ресурса, но сохраняется требование к качеству предоставления ТУС [10]. Следовательно, задачу моделирования может быть сформулирована в условиях обеспечения приоритета сообщений ТУС (ПБД туС) относительно сообщений в модальном представлении (ПБДмод). Однако, вследствие необходимости синхронизации модальностей, требуется обеспечить своевременную доставки ПБДмод без изменения взаимных временных соотношений между ними, иначе достоверность суждения о лигитимности и девиантности операторов будет неудовлетворительной. Соответственно, предлагаемая модель в формализованном виде может быть представлена как 
система массового обслуживания с РКР следующим образом.

Дано: система массового обслуживания (СМO), В которой вектор параметров входной нагрузки вида $\vec{Z}_{k}=\left[b_{k}, N_{k}, \alpha_{k}, \mu_{k}\right]^{T}$, где $k=1, \ldots, n, n-$ число потоков заявок, $b_{k}$ - число единиц канального ресурса (ЕКР) линии, необходимого для обслуживания $k$-го потока; $\alpha_{k}$ - интенсивность потока заявок от одного источника $k$-го приоритета в свободном состоянии; $\mu_{k}-$ интенсивность обслуживания заявок $k$-го потока; $N_{k}$ - общее число источников, формирующих $k$-й поток нагрузки; $\varphi_{k}(i)$ - градиентная функция внутренней блокировки для заявок $k$-го потока, где $i-$ общее число занятых канальных единиц объема канального ресурса $V$ в момент поступления заявки $k$-го потока; $V$ - объем канального ресурса.

Дисциплина обслуживания - с абсолютным приоритетом и РКР.

Ограничения и допущения: процесс обслуживания заявок рассматривается на уровне соединения; поступающие входные потоки описываются моделью простейшего потока с параметром $\alpha_{k}\left(N_{k}<100\right)$; длительность обслуживания заявок потоков имеет экспоненциальное распределение с параметром $\mu_{k}$; функция внутренней блокировки $\varphi_{k}(i) \neq 1$ и является неубывающей.

Необходимо: разработать математическую модель системы, учитывающую дисциплину обслуживания заявок с приоритетами, прерыванием и резервированием канального ресурса, определяющую зависимость

$$
\pi_{k}=F\left(\stackrel{1 !}{Z_{k}}, V, n\right),
$$

где $\pi_{k}$ - показатель качества обслуживания в виде вероятности потерь заявок $k$-го потока.

В символике Кендалла-Башарина модель СМО представляется в виде

$$
\stackrel{!}{M i_{n}} / \stackrel{4}{M} / V / L / P R A
$$

Ниже раскрываются элементы этого представления.

1. Входной поток - $M i_{n}$. На СМО поступает $n$ примитивных потоков заявок (вызовов) с общим числом источников $N_{k}, k=1, \ldots, n ; N_{k}<100$.

2. Поток освобождений - $M_{l}$. Среднее время занятия канального ресурса обслуживанием одной заявки $k$-го потока равно $1 / \mu_{k}$. Число ЕКР, необходимое для обслуживания одной заявки $k$-го потока, - $b_{k}$ (целое число). Длительности обслуживания заявок $k$-го потока являются случайной величиной, имеют экспоненциальное распреде- ление и не зависят друг от друга и от других входных потоков.

3. Скорость передачи информации в канале связи, выраженная в ЕКР,- $V$ (целое число).

4. Способ обслуживания с явными потерями $-L$.

5. Порядок обслуживания с абсолютным приоритетом - PRA.

Моделирование звена СПД системы мониторинга КВО. Пусть на звено СПД со скоростью передачи информации $V$ поступают два потока заявок $(n=2)$, ПБДмод и ПБД далее обозначается через $\pi_{k}$. Заявки второго потока $(k=$ 2) обладают абсолютным приоритетом по отношению к заявкам первого потока $(k=1)$ за исключением случаев, предусмотренных РКР для низкоприоритетных заявок с характеристиками субъекта контроля (оператора APM).

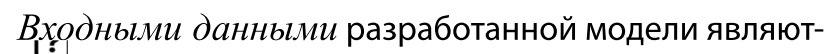
ся: $Z_{k}$ - вектор параметров входной нагрузки, определяемый как

$$
\vec{Z}_{k}=\left[b_{k}, N_{k}, \alpha_{k}, \mu_{k}\right]^{T} .
$$

Bblходныле данныле модели: $\pi_{1}, \pi_{2}$.

Число заявок первого и второго потока, находящихся на обслуживании, далее обозначается в виде

$$
i_{1} \in\{0,1, \ldots,] \frac{V}{b_{1}}[\} \text { и } i_{2} \in\{0,1, \ldots,] \frac{V}{b_{2}}[\}
$$

соответственно, где скобки ].[ обозначают целую часть от соответствующего выражения. Общее число занятых ЕКР определяется из соотношения $i=i_{1} b_{2}+i_{2} b_{2}$. Тогда вектор $\left(i_{1}, i_{2}\right)$ отражает состояние системы.

При поступлении высокоприоритетного вызова возможны исходы:

вызов будет принят на обслуживание с вероятностью $1-\varphi_{2}(i)$, при $\varphi_{2}(i) \in[0 ; 1)$, не влияя на число установленных низкоприоритетных соединений. Ситуация возможна, когда число свободных ресурсов звена больше или равна $b_{2}$ EKР, т.е. при $V-i \geq b_{2}$;

вызов будет принят на обслуживание с вероятностью $1-\varphi_{2}(i)$, при $\varphi_{2}(i) \in[0 ; 1)$ за счет прерывания

$$
\left\lceil\frac{b_{2}-(V-i)}{b_{1}}\right\rceil
$$

установленных низкоприоритетных соединений, где $\lceil\cdot\rceil$ - округление к большему целому. Ситуация возмож- 


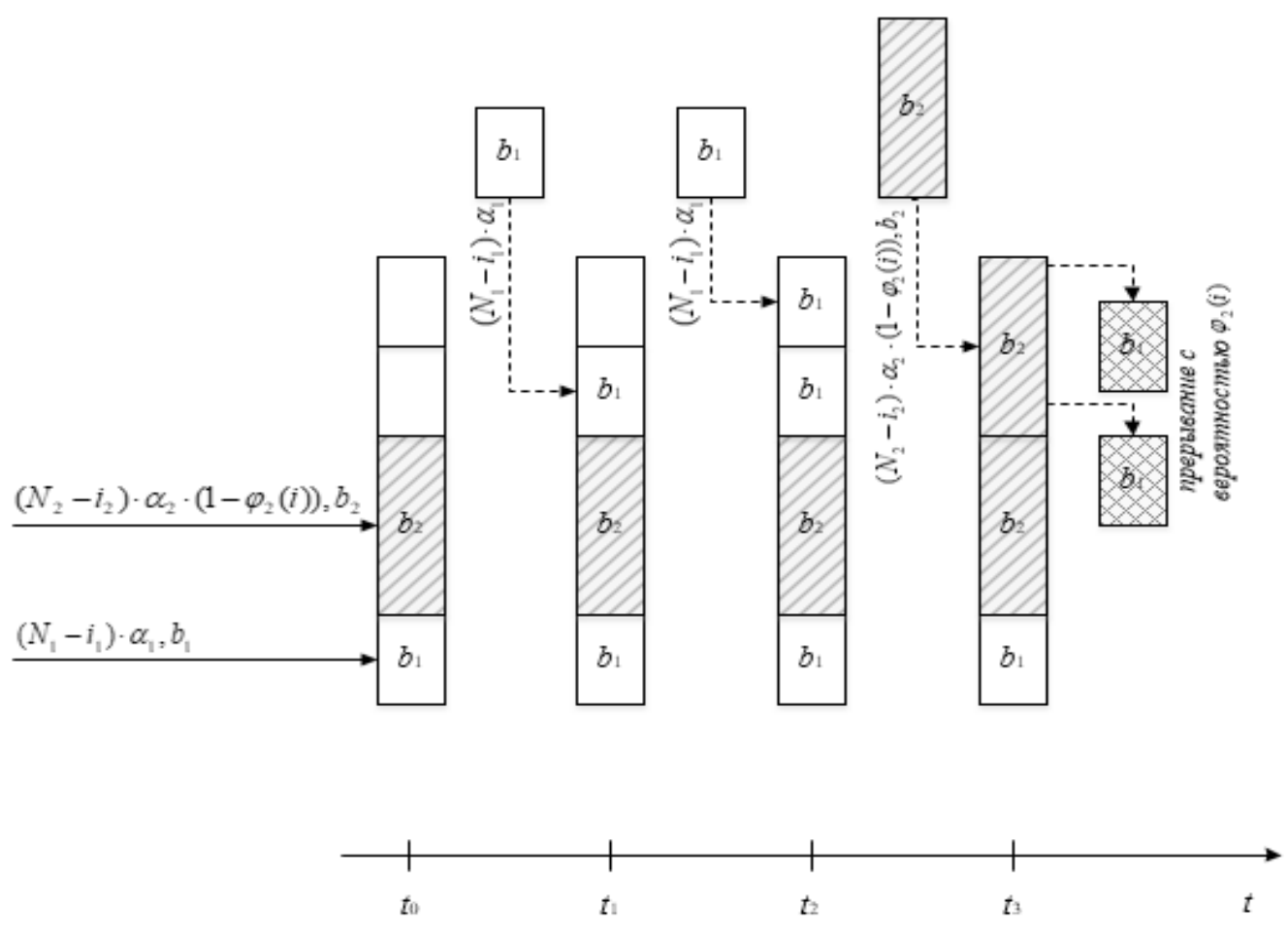

Рис. 1. Реализация механизма прерывания установленных низкоприоритетных соединений при использовании функции внутренней блокировки

на, когда число свободных ресурсов звена меньше числа EKP $b_{2}$, требуемых для установления высокоприоритетного соединения, т.е. при $V-i<b_{2}$; вызов будет потерян, когда число ресурсов звена, не занятых обслуживанием высокоприоритетных соединений, меньше числа EKP $b_{2}$, т.е. при $V-i_{2} b_{2}<b_{2}$.

При поступлении низкоприоритетного вызова возможны исходы: вызов будет принят на обслуживание. Ситуация возможна, когда число свободных ресурсов звена больше или равно $b_{1}$ единиц канального ресурса, т.е. при $V-i \geq b_{1}$; вызов будет потерян во всех остальных случаях.

Описанные правила приема и обслуживания вызовов показаны на рисунке 1 для случая, когда $b_{1}=1, b_{2}=2$, $V=5$.

Сформулированная постановка задачи определяет вид пространства состояний исследуемой двухпотоковой модели звена СПД и структуру случайного процесса, описывающего динамику их изменения. Через $i_{l}(t)$ далее обозначается число заявок первого $(k=1)$ и $i_{2}(t)$ - второго $(k=2)$ потоков, находящихся в момент времени $t$ на обслуживании. Динамика изменения с течением времени числа обслуживаемых заявок каждого из имеющихся потоков описывается двумерным случайным процессом $r(t)=\left(i_{1}(t), i_{2}(t)\right)$, определённым на конечном пространстве состояний $\Omega=\left\{\left(i_{1}, i_{2}\right): i \leq V\right\}$. Пространство состояний и соответствующая диаграмма переходов CMO, рассматриваемого типа, показаны на рисунке 2.

\section{Характеристики качества обслуживания вызовов}

Приведенная постановка задачи и математическое описание модели позволяют утверждать, что процесс $r(t)$ является марковским и может быть исследован в стационарном режиме. Пусть $P(i 1, i 2)-$ стационарная вероятность того, что на обслуживании находятся і1 заявок на передачу низкоприоритетного трафика ПБД и $і 2$ заявок высокоприоритетного трафика ПБД

В соответствии с интерпретацией стационарных вероятностей марковского процесса $r(t)$ значение вероятности $P\left(i_{1}, i_{2}\right)$ представляет собой долю времени пребывания звена в состоянии $\left(i_{1}, i_{2}\right)$.

Приведенная интерпретация позволяет определить искомые характеристики качества обслуживания поступающих потоков заявок. К ним относятся вероятности потери заявок на передачу низкоприоритетного трафика $\pi_{l}$ сообщений в модальном представлении и высокоприоритетного трафика $\pi_{2}$ ТУС. 


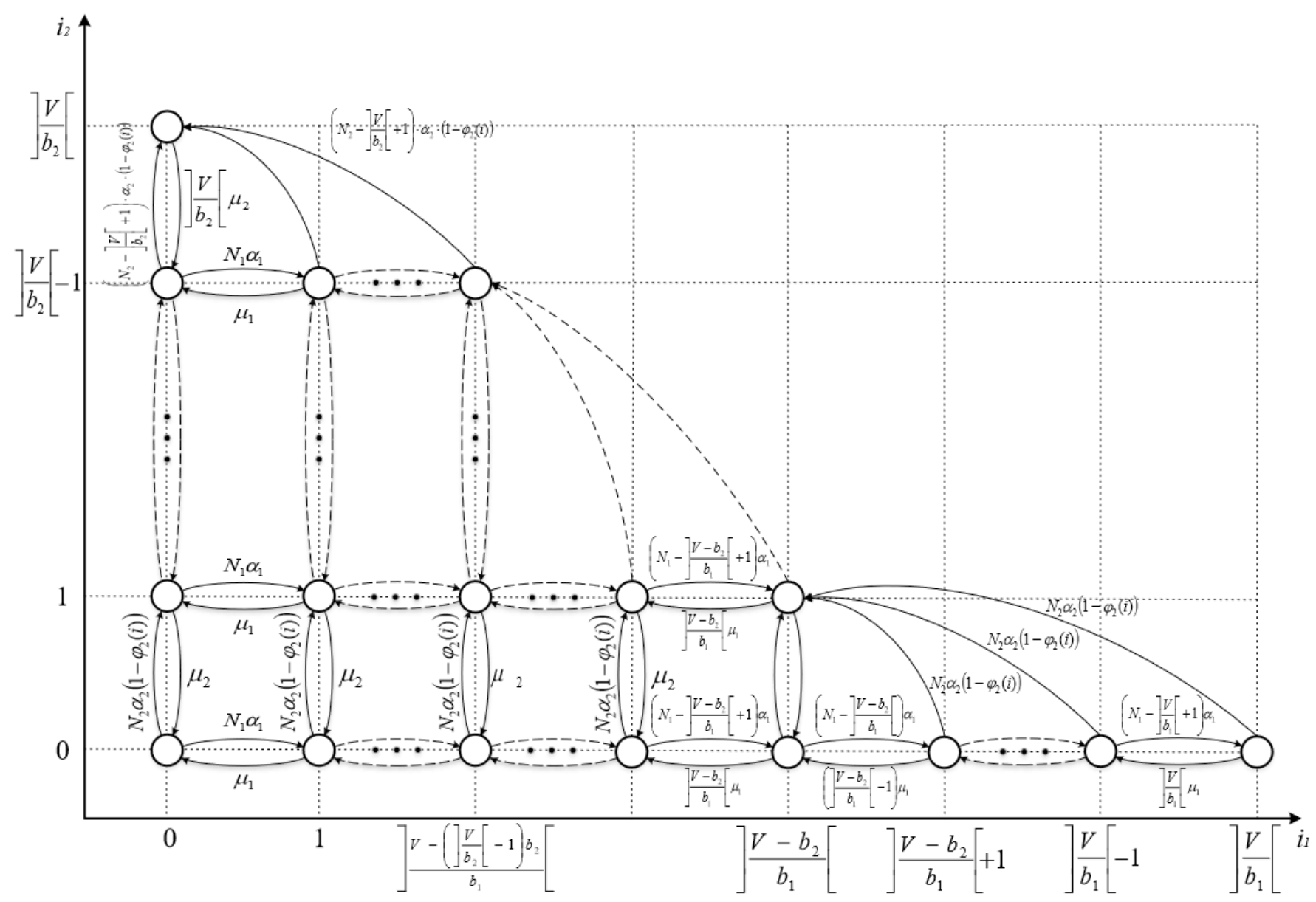

Рис. 2. Диаграмма переходов для случайного процесса r(t)

Ниже приводится общие выражения и порядок оценивания данных показателей $\pi_{1}=\pi+\pi$, где $\pi_{\text {пт }}$ вероятность потерь низкоприоритетного трафика из-за занятости всего канального ресурса обслуживанием заявок обоих потоков, определяемая как отношение интенсивности потерянных заявок к интенсивности поступивших заявок

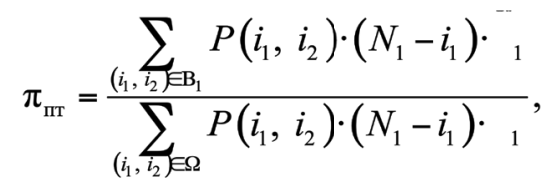

где $\mathrm{B}_{1}=\left\{\left(i_{1}, i_{2}\right) \in \Omega: i+b_{1}>V\right\} ; \pi_{\text {пр }}$ - вероятность потерь низкоприоритетного трафика из-за прерывания соединения заявкой более высокого приоритета, определяемая как отношение интенсивностей соответствующих событий. Высокоприоритетная заявка будет принята к обслуживанию с вероятностью $1-\varphi_{2}(i)$, если компоненты состояния СМО удовлетворяют условию $i_{2} \cdot b_{2}+b_{2} \leq V$.
Прерывание низкоприоритетных заявок для компонент рассматриваемого состояния $\left(i_{1}, i_{2}\right)$ происходит при выполнении неравенства $i+b_{2}>V$. В этом случае число прерываемых заявок будет определяться из выражения

$$
\begin{aligned}
& \left\lceil\frac{b_{2}-(V-i)}{b_{1}}\right]: \\
& \pi_{\mathrm{mp}}=\frac{\sum_{\left(i_{1}, i_{2}\right) \in B_{2}}\left(\left[\frac{b_{2}-(V-i)}{b_{1}}\right]\right) \cdot P\left(i_{1}, i_{2}\right) \cdot\left(N_{2}-i_{2}\right) \cdot{ }_{2} \cdot\left(-{ }_{2} i\right)}{\sum_{\left(i_{1}, i_{2}\right) \in \Omega} P\left(i_{1}, i_{2}\right) \cdot\left(N_{1}-i_{1}\right) \cdot 1_{1}},
\end{aligned}
$$

где $\mathrm{B}_{2}=\left\{\left(i_{1}, i_{2}\right) \in \Omega: V-i_{1} \cdot b_{1}<i_{2} \cdot b_{2}+b_{2} \leq V\right\}$;

$\pi_{2}$ - вероятность потерь высокоприоритетного трафика из-за занятости всего канального ресурса и с учетом РКР определяется выражением $\pi_{2}=\pi_{\Pi т 2}+\pi_{\phi \sigma}$, где $\pi_{\text {Пт2 }}$ - вероятность потерь высокоприоритетного трафика ТУС из-за занятости всего канального ресурса обслуживанием заявок приоритетного потока, определя- 
емая как отношение интенсивности потерянных заявок к интенсивности поступивших заявок

$$
\pi_{n m 2}=\frac{\sum_{\left(i_{1}, i_{2}\right) \in \mathrm{B} 3} P\left(i_{1}, i_{2}\right) \cdot\left(N_{2}-i_{2}\right) \cdot\left(1-\varphi_{2}(i)\right)}{\sum_{\left(i_{1}, i_{2}\right) \in \Omega} P\left(i_{1}, i_{2}\right) \cdot\left(N_{2}-i_{2}\right) \cdot\left(1-\varphi_{2}(i)\right)}, \text { (3) }
$$

где $\mathrm{B}_{3}=\left\{\left(i_{1}, i_{2}\right) \in \Omega: i_{2} \cdot b_{2}+b_{2}>V\right\} ; \pi_{\phi \sigma}$ - вероятность потерь высокоприоритетного трафика из-за функции блокировки:

$$
\pi_{\phi \sigma}=\sum_{\left(i_{1}, i_{2}\right) \in \Omega} P\left(i_{1}, i_{2}\right) \cdot \varphi_{2}(i) .
$$

Приведенные определения показателей качества обслуживания заявок и заданная функция блокировки для высокоприоритетного трафика ТУС дают возможность произвести оценку отличий предложенной модели СПД как СМО с РКР относительно известных моделей с отказами и абсолютным приоритетом.

\section{Система \\ уравнений равновесия}

\section{Для оценивания характеристик модели СМО}

$$
\stackrel{!}{M i_{n}} / \frac{L_{M}}{M} / V / L / P R A
$$

необходимо составить и решить систему уравнений равновесия (СУР), связывающую значения стационарных вероятностей $P\left(i_{1}, i_{2}\right)$.

В соответствии с правилом статистического равновесия при формировании системы уравнений необходимо определить и просуммировать интенсивности поступления всех событий, которые выводят процесс $r(t)$ из произвольного состояния $\left(i_{1}, i_{2}\right)$, взвешенные вероятностью данного состояния (левая часть (УР) и приравнять их к суммарным интенсивностям перехода процесса $r(t)$ в состояние $\left(i_{1}, i_{2}\right)$, взвешенным соответствующими вероятностями состояний, из которых этот переход происходит (правая часть СУР) $[6,9]$.

Сначала целесообразно получить выражение для левой части произвольного уравнения системы уравнений равновесия. Переход из состояния $\left(i_{1}, i_{2}\right)$ осуществляется при наступлении следующих событий.

1. При поступлении заявки на передачу низкоприоритетного трафика. Данное событие наступает с интенсивностью $\left(N_{1}-i_{1}\right) \cdot a_{1}$ при условии, что есть свободный канальный ресурс для обслуживания поступившей заявки (т.е. при $i+b_{1} \leq V$ ). В этом случае совершается переход в состояние $\left(i_{1}+1, i_{2}\right)$.
2. При поступлении заявки на передачу высокоприоритетного трафика. Данное событие наступает с интенсивностью $\left(N_{2}-i_{2}\right) \cdot \mathrm{a}_{2} \cdot\left(1-\varphi_{2}(i)\right)$ при условии, что механизм допуска заявок принимает решение о ее приеме на обслуживание и функция блокировки $\varphi_{2}(i) \neq 1$. Указанное действие выполняется в двух случаях:

если есть свободный канальный ресурс для обслуживания поступившей заявки, т.е. $i+b_{2} \leq V$. В этом случае совершается переход в состояние $\left(i_{1}, i_{2}+1\right)$;

если число свободных ресурсов звена меньше $b_{2}$, т.е. $V-i<b_{2}$. В этом случае происходит прерывание обслуживания

$$
\left\lceil\frac{b_{2}-(V-i)}{b_{1}}\right\rceil
$$

низкоприоритетных соединений, и совершается переход в состояние

$$
\left(i_{1}-\left(\left\lceil\frac{b_{2}-(V-i)}{b_{1}}\right\rceil\right), i_{2}+1\right) \text {. }
$$

3. При окончании обслуживания заявки на передачу низкоприоритетного трафика. Данное событие наступает с интенсивностью $i_{1} \cdot \mu_{1}$, если на обслуживании имеются соответствующие заявки $\left(i_{1}>0\right)$. В этом случае совершается переход в состояние $\left(i_{1}-1, i_{2}\right.$.

4. При окончании обслуживания заявки на передачу высокоприоритетного трафика. Данное событие наступает с интенсивностью $i_{2} \cdot \mu_{2}$, если на обслуживании имеются соответствующие заявки $\left(i_{2}>0\right)$. В этом случае совершается переход в состояние $\left(i_{1}, i_{2}-1\right)$.

Правая часть произвольного уравнения СУР отражает, из каких состояний, с какой интенсивностью и при каких условиях возможен переход процесса $r(t)$ в заданное состояние $\left(i_{1}, i_{2}\right)$. Интересны в этом контексте соответствующие события и интенсивности их осуществления.

Переход случайного процесса $r(t)$ в состояние $\left(i_{1}, i_{2}\right)$ может произойти в следующих ситуациях:

1. Из состояния $\left(i_{1}-1, i_{2)}\right.$ с интенсивностью $\left(N_{1}-i_{1}+\right.$ 1) $a_{l}$ в результате поступления заявки на передачу низкоприоритетного трафика. Условием осуществления события является принадлежность состояния $\left(i_{1}-1, i_{2}\right)$ пространству состояний модели $\Omega$

$$
\left(\text { т. е. при } 0<i_{1} \leq\right] \frac{V-i_{2} \cdot b_{2}}{b_{1}}[) \text {. }
$$




$$
\begin{gathered}
P\left(i_{1}, i_{2}\right) \cdot\left[\left(N_{1}-i_{1}\right) \cdot{ }_{1} \cdot I\left(i+b_{1} \leq V\right)+\left(N_{2}-i_{2}\right) \cdot{ }_{2} \cdot\left(-{ }_{2} i\right) \cdot I\left(i+b_{2} \leq V\right)+\right. \\
\left.+\left(N_{2}-i_{2}\right) \cdot \alpha_{2} \cdot\left(1-\varphi_{2}(i)\right) \cdot I\left(i+b_{2}>V, i_{2} \cdot b_{2}+b_{2} \leq V\right)+\sum_{k=1}^{2} i_{k} \cdot \mu_{k} \cdot I\left(i_{k}>0\right)\right]= \\
=P\left(i_{1}-1, i_{2}\right) \cdot\left(N_{1}-i_{1}+1\right) \cdot{ }_{1} \cdot I\left(<i_{1} \leq \frac{V-i_{2} \cdot b_{2}}{b_{1}}[)+\right. \\
+P\left(i_{1}, i_{2}-1\right) \cdot\left(N_{2}-i_{2}+1\right) \cdot{ }_{2} \cdot\left(-{ }_{2} i-b_{2}\right) \cdot I\left(<i_{2} \leq\right] \frac{V-i_{1} \cdot b_{1}}{b_{2}}[)+ \\
+P\left(i_{1}+1, i_{2}\right) \cdot\left(i_{1}+1\right) \cdot{ }_{1} \cdot I\left(i+b_{1} \leq V\right)+P\left(i_{1} i_{2}+\right) \cdot\left(i_{2}+\right) \cdot{ }_{2} \cdot I\left(i+b_{2} \leq V\right)+ \\
=P\left(i_{1}-1, i_{2}\right) \cdot\left(N_{1}-i_{1}+1\right) \cdot{ }_{1} \cdot I\left(<i_{1} \leq\right] \frac{V-i_{2} \cdot b_{2}}{b_{1}}[)+ \\
+P\left(i_{1}, i_{2}-1\right) \cdot\left(N_{2}-i_{2}+1\right) \cdot{ }_{2} \cdot\left(-{ }_{2} i-b_{2}\right) \cdot I\left(<i_{2} \leq\right] \frac{V-i_{1} \cdot b_{1}}{b_{2}}[)+ \\
+P\left(i_{1}+1, i_{2}\right) \cdot\left(i_{1}+1\right) \cdot{ }_{1} \cdot I\left(i+b_{1} \leq V\right)+P\left(i_{1} i_{2}+\right) \cdot\left(i_{2}+\right) \cdot{ }_{2} \cdot I\left(i+b_{2} \leq V\right)+ \\
\cdot I\left(i_{2}>0, i_{2} \cdot b_{2} \leq V, i+b_{1}>V\right),\left(i_{1}, i_{2}\right) \in \Omega .
\end{gathered}
$$

Здесь $I(\cdot)$ - индикаторная функция, определяемая соотношением

$$
I(\cdot)=\left\{\begin{array}{l}
1, \text { если выполнено условие, сформулированное в скобках; } \\
0, \text { если это условие не выполнено. }
\end{array}\right.
$$

2. В результате поступления заявки на передачу высокоприоритетного трафика, если механизм допуска заявок принимает решение о ее приеме на обслуживание. Указанное действие выполняется в двух случаяx:

из состояния $\left(i_{1}, i_{2}-1\right)$ с интенсивностью $\left(N_{2}-i_{2}+\right.$ 1). $a_{2} \cdot\left(1-\varphi_{2}(i)\right)$. Условием осуществления события является принадлежность состояния $\left(i_{1}, i_{2}-1\right)$ пространству состояний модели $\Omega$

$$
\left(\text { т. е. при } 0<i_{2} \leq\right] \frac{V-i_{1} \cdot b_{1}}{b_{2}}[) \text {; }
$$

из состояний $\left(i_{1}+k, i_{2}-1\right)$, где

$$
k=1, \overline{\frac{V-\left(i_{2}-1\right) \cdot b_{2}}{b_{1}}\left[-i_{1}\right.},
$$

с интенсивностью $\left(N_{2}-i_{2}+1\right) \cdot a_{2} \cdot\left(1-\varphi_{2}(i)\right)$, в случае прерывания низкоприоритетной заявки. Условием осуществления события является принадлежность состоя- ний $\left(i_{1}+k, i_{2}-1\right)$ пространству состояний модели $\Omega$ (т.е. при $i_{2}>0$ и $i_{2} \cdot b_{2} \leq V, i+b_{1}>V$ ).

3. Из состояния $\left(i_{1}+1, i_{2}\right)$ с интенсивностью $\left(i_{1}+1\right) \cdot \mu_{1}$ в результате окончания обслуживания заявки на передачу низкоприоритетного трафика. Условием осуществления события является принадлежность состояния $\left(i_{1}+1, i_{2}\right)$ пространству состояний модели $\Omega$ (т.е. при $i+b_{1} \leq V$ ).

4. Из состояния $\left(i_{1}, i_{2}+1\right)$ с интенсивностью $\left(i_{2}+1\right) \cdot \mu_{2}$ в результате окончания обслуживания заявки на передачу высокоприоритетного трафика. Условием осуществления события является принадлежность состояния $\left(i_{1}\right.$, $\left.i_{2}+1\right)$ пространству состояний модели $\Omega$ (т.е. при $i+b_{2}$ $\leq V)$.

Приравняв левую и правую части уравнения равновесия можно получить общий вид СУР (5).

Следует отметить, что решение системы уравнений равновесия (5) удовлетворяет условию нормировки $\sum_{\left(i_{1}, i_{2}\right) \in \Omega} P\left(i_{1}, i_{2}\right)=1$. 


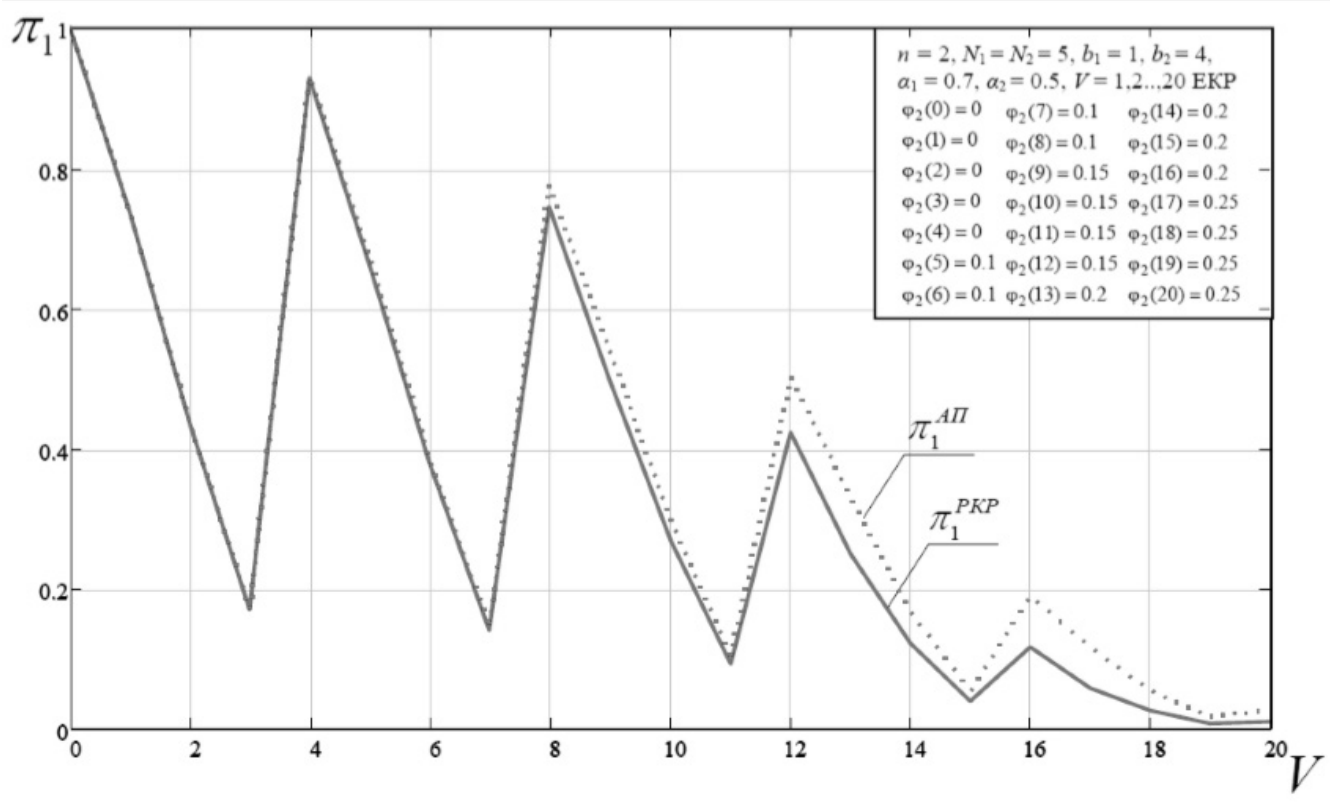

Рис. 3. Зависимость вероятности потерь низкоприоритетных заявок от канального ресурса известной и предлагаемой модели: $\pi_{l}^{A \Pi}$ — вероятности потерь низкоприоритетных заявок известной модели, $\pi_{l}^{P К P}$ — вероятности потерь низкоприоритетных заявок предлагаемой модели с учетом РКР.

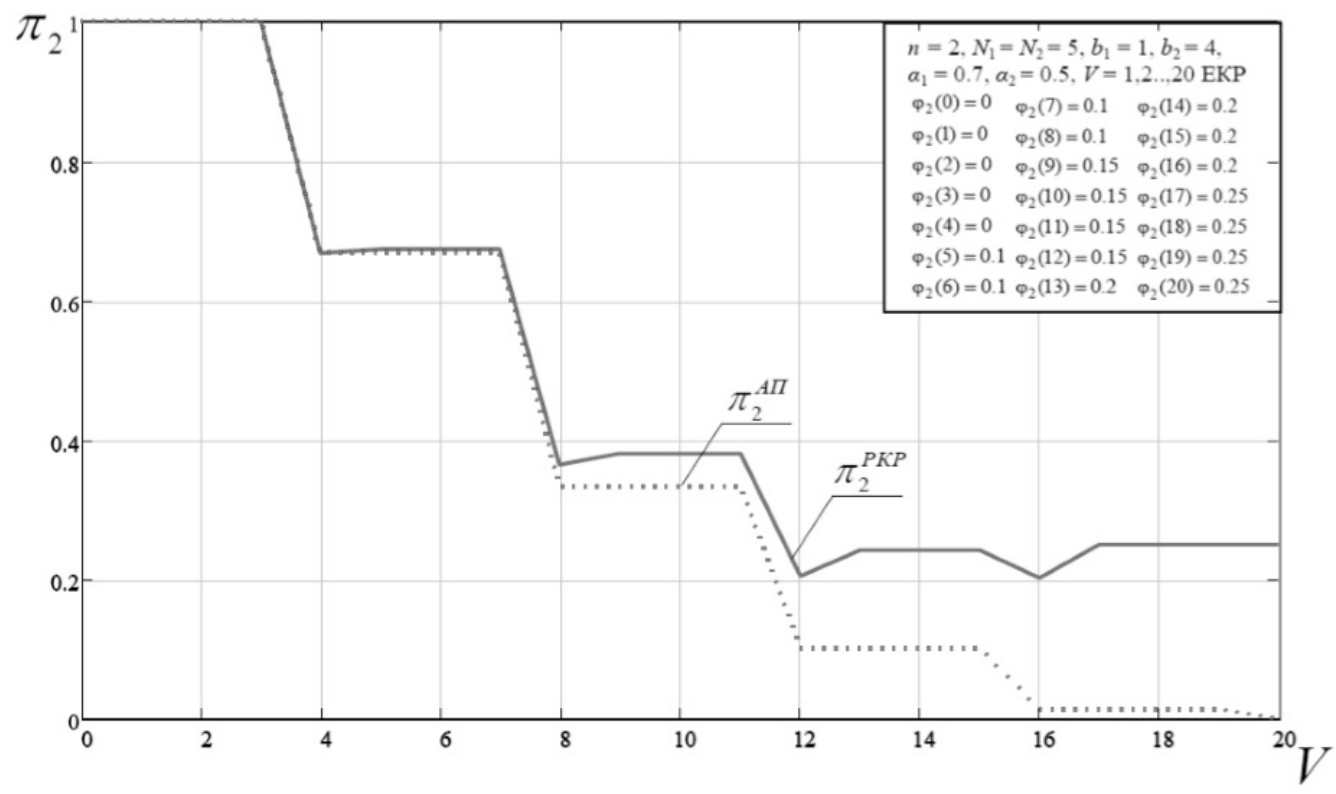

Рис. 4. Зависимость вероятности потерь высокоприоритетных заявок от канального ресурса известной и предлагаемой модели: $\pi_{2}^{A I}$ - вероятности потерь высокоприоритетных заявок существующей модели, $\pi_{2}{ }^{P K P}$ - вероятности потерь высокоприоритетных заявок предлагаемой модели с учетом РКР.

В связи с реализацией механизма прерывания для данной модели не выполняется критерий Колмогорова, следовательно, процесс $r(t)$ не обладает свойством обратимости. Поэтому распределение вероятностей состояний системы не может быть представлено в мульти- пликативном виде [11, 12]. В связи с этим для решения СУР (5) необходимо использовать численные методы. В этом случае рекомендуется использовать итерационный метод Гаусса-Зейделя, общая схема реализации которого при решении СУР представлена в [13]. 
В связи с предложенной концепцией, предлагается, варьируя значения вероятностей отказа в обслуживании высокоприоритетного трафика в зависимости от общего числа занятых ЕКР, исследовать алгоритмы резервирования канального ресурса СПД (рисунки 3 и 4). Наблюдаемое локальное уменьшение вероятности потерь низкоприоритетных заявок объясняется вытеснением с обслуживания ресурсоемких заявок в соответствии с правилом, заданным функцией внутренней блокировки.

Представленный механизм позволяет обеспечить как необходимое качество обслуживания сообщений ПБДМОД, так и соответствующее качество ТУС в зависимости от актуальной обстановки по связи.

Моделирование обеспечило описание закономерностей зависимости качества обслуживания ПБД тус и ПБД (+БД). Данная модель позволяет разработать алгоритм для определения оптимальных значений управляемых характеристик звена СПД, а также решить задачу, заключающуюся в поиске таких функций блокировок СПИ, при которых вероятность потерь низкоприори- тетных заявок ПБДмод будет минимальной, а показатели качества обслуживания ПБД рамках.

\section{Зак^ючение}

Таким образом, для системы мониторинга КВО с реализованным контролем состояния операторов АРМ на базе ДМА предложена новая математическая модель $\mathrm{CMO}$

$$
\stackrel{4}{M i_{n}} / \stackrel{\breve{L}}{M} / V / L / P R A
$$

с градиентным резервированием канального ресурса вида

$$
\pi_{k}=F\left(\stackrel{1 !}{Z_{k}}, V, n\right)
$$

Предложенная модель в комплексе с известными моделями систем распределения информации [12-15] позволит решать оптимизационные задачи, связанные с определением наиболее предпочтительных параметров при проектировании СПД системы мониторинга КВО государства.

\section{ЛИТЕРАТУРА}

1. Федеральный закон РФ 68-Ф3 от 21.12.1994 (с изменениями от 8.03.2015) «0 защите населения и территорий от чрезвычайных ситуаций природного и техногенного характера» / М.: Собрание законодательства РФ, 2017.

2. Федеральный закон РФ N187-Ф3 от 26.07.2017 «0 безопасности критической информационной инфраструктуры Российской Федерации» / М.: Собрание законодательства РФ, 2017.

3. Постановление Правительства Российской Федерации от 14 августа 2020 г. № 1225 «06 утверждении правил разработки критериев отнесения объектов всех форм собственности к критически важным объектам»/ https://www.garant.ru/products/ipo/prime/doc/74423898.

4. Саитов И. А. Методологические основы синтеза полимодальных инфокоммуникационных систем государственного управления: монография / И. А. Саитов, 0. О. Басов, А. А. Карпов, — Орёл: Академия ФСО России, 2015.— 263 с.

5. Носов М. В. Повышение эффективности управления в условиях изменения психофизиологического состояния персонала / М. В. Носов, О. О. Басов П. Ю. Хахамов // Труды СПИИРАН.— 2014.—- Вып. 3 (34).—C. 112-135.

6. Клейнрок Л. Теория массового обслуживания / Л. Клейнрок - М.: Машиностроение, 1979-432 с.

7. Evans J., Filsfils C. Deploying IP and MPLS QoS for Multiservice Networks. Theory and Practice, Morgan Kaufmann Publishers, 2007, 456 p.

8. Степанов С. Н. Основы телетрафика мультисервисных сетей / С. Н. Степанов.— М.: Эко-Трендз, 2010.— 392 с.

9. Саитов И. А. Теоретические основы анализа и оптимизации иерархических многоуровневых маршрутизирующих систем: монография / И. А. Саитов Р. Б. Трегубов. — Орёл: Академия ФСО России, 2017. — 587 с.

10. Концепция управления качеством связи в Российской Федерации (проект). - М.: Министерство связи и массовых коммуникаций Российской Федерации, 2015.

11. Iversen V. B. Teletraffic Engineering and Network Planning. DTU Fotonik, 2015, $382 \mathrm{p}$.

12. Наумов В. А. Теория телетрафика мультисервисных сетей. - М., РУДН, 2007. - 191 с.

13. Степанов С. Н. Теория телетрафика: концепции, модели, приложения. М., Горячая линия, Телеком, 2015, 868 с. 